\section{Pomalidomid bei Myelom noch in scheinbar aussichtslosen Situationen von Nutzen}

\author{
Immunmodulatorische Substanzen haben die Prognose beim multiplen \\ Myelom (MM) verbessert. Doch nach Rezidiv ist das Überleben weiterhin \\ schlecht. Auch in dieser Situation ist Pomalidomid noch aktiv.
}

P atienten mit rezidiviertem multiplem Myelom (MM) haben eine schlechte Prognose. Vor allem bei Refraktärität gegen Immunmodulatoren der 1 . Generation wie Thalidomid und Lenalidomid oder gegen den Proteasominhibitor Bortezomib sind die Optionen für die stark vorbehandelten, häufig komorbiden und von Nebenwirkungen geschwächten $\mathrm{Pa}$ tienten limitiert. Tolerabel in dieser $\mathrm{Si}$ tuation ist Pomalidomid. Der Immunmodulator der 2. Generation ist auch nach Zweit-, Dritt- und Viertlinien-Rediziven aktiv und verlängert das mediane Gesamtüberleben im Vergleich zu historischen Kontrollen weiter.

Das Potenzial von Pomalidomid bei Patienten mit stark fortgeschrittenem, rezidiviertem sowie refraktärem MM (RRMM) wurde in der multizentrischen Phase-II-Studie IFM2009-02 untersucht. In dieser wurde Pomalidomid zusammen mit niedrig dosiertem Dexamethason bei 84 Patienten mit sehr fortgeschrittenem MM täglich verabreicht. Diese Medikation verlängerte bei rund $40 \%$ der Patienten progressionsfreies (PFS) und Gesamtüberleben (OS).

Die Patienten wurden in 2 Gruppen aufgeteilt: Eine Gruppe hatte den Immunmodulator 3 Monate bis zu maximal 1 Jahr erhalten, die andere Gruppe mehr als 1 Jahr. Bei den kürzer als 1 Jahr behandelten Patienten betrug die Gesamtansprechrate $43 \%$, das mediane PFS 4,6 Monate (95\%-Konfidenzinter- vall [95\%-KI] I 3,8-6,4) und das mediane OS 15 Monate (95\%-KI 11,7-20,3). $40 \%$ der Patienten lebten nach 1,5 Jahren noch. Hatten die Patienten Pomalidomid länger als ein Jahr erhalten, ergaben sich überraschenderweise deutlich bessere Werte: Die Gesamtansprechrate lag bei $83 \%$ und das mediane PFS bei 20,2 Monaten (95\%-KI 14,7-35,4). Das mediane OS war zum Analysezeitpunkt noch nicht erreicht, die OS-Rate betrug nach 18 Monaten $91 \%$.

Fazit: Der Immunmodulator Pomalidomid plus Dexamethason verlängert das Überleben von RRMM-Patienten weit über die bisher bekannten Zeiten hinaus. Interessant sind vor allem die „Long runners on pomalidomide": Bei ihnen wird das Ergebnis um so besser, je länger die Behandlung dauert. Weitere Studien werden diesen ersten Eindruck bestätigen müssen.

Barbara Kreutzkamp

Fouquet $\mathrm{G}$ et al. Safe and prolonged survival with long-term exposure to pomalidomide in relapsed/refractory myeloma. Ann Oncol. 2016;27(5):902-7.

\section{Carfilzomib: Hoffnung beim refraktären Myelom}

\author{
Für Patienten mit therapierefraktärem multiplem Myelom ist der \\ Proteasominhibitor Carfilzomib in Kombination mit Dexamethason eine \\ vielversprechende Alternative. In einer Phase-III-Studie wurde die Substanz \\ gegen einen Therapiestandard getestet; erste Ergebnisse liegen jetzt vor.
}

\footnotetext{
$\mathrm{n}$ der Therapie des rezidivierten oder refraktären Myeloms (RRMM) gilt der Proteasominhibitor Bortezomib in Kombination mit Dexamethason als ein Standard. Carfilzomib, ein weiterer Proteasominhibitor, hat in Kombination mit Dexamethason vielversprechende Aktivität gezeigt. Nun wurde in der Phase-III-Studie ENDEAVOR die Kombination Bortezomib plus Dexamethason mit der Kombination Carfilzomib plus Dexamethason verglichen. Die 929 Teilnehmer mit RRMM hatten bereits bis zu 3 erfolglose Therapieversuche hinter sich. 465 Patienten erhielten Bortezomib/ Dexamethason, 464 bekamen Carfilzo$\mathrm{mib} /$ Dexamethason. Eine geplante Interimsanalyse wurde nach 414 Ereignissen
}

(Tod oder Krankheitsprogression) vorgenommen; 171 Fälle waren in der Carfilzomib-, 243 in der Bortezomib-Gruppe aufgetreten. Mit einem medianen progressionsfreien Überleben von 18,7 gegenüber 9,4 Monaten war die Carfilzomib-Kombination der Standardtherapie im primären Endpunkt deutlich überlegen (Hazard Ratio [HR] 0,53, $95 \%$-Konfidenzintervall $0,44-0,65$; $\mathrm{p}<0,0001)$. Carfilzomib punktete auch bei der objektiven Ansprechrate (77 vs. $63 \%$; Odds Ratio [OR] 2,03; p < 0,0001) und der Dauer des Ansprechens (21,3 vs. 10,4 Monate).

Insgesamt waren unter Carfilzomib schwere Nebenwirkungen häufiger (48 vs. $36 \%$ ). Zu den häufigsten Nebenwir- kungen vom Grad $\geq 3$ zählten Anämie (14 vs. $10 \%$ ), Bluthochdruck (9 vs. $3 \%$ ), Thrombozytopenie (8 vs. $9 \%$ ) und Pneumonie (7 vs. $8 \%$ ). Bei $23 \%$ gegenüber $48 \%$ der Patienten musste die Dosis aufgrund von Nebenwirkungen reduziert werden. Dabei ging die Dosisreduktion in der Bortezomib-Gruppe in $62 \%$ der Fälle auf eine Neuropathie zurück, in der Carfilzomib-Gruppe nur in $7 \%$.

Fazit: Die Daten der ENDEAVOR-Studie liefern starke Evidenz zugunsten der Behandlungsstrategie mit Carfilzomib. Allerdings profitierten Patienten, die mit Lenalidomid vorbehandelt waren und auf dieses nicht angesprochen hatten, nicht maßgeblich von der CarfilzomibKombination. Zudem traten akute Nierenschäden unter Carfilzomib bei $8 \%$ der Patienten auf.

Elke Oberhofer

Dimopoulos MA et al. Carfilzomib and dexamethasone versus bortezomib and dexamethasone for patients with relapsed or refractory multiple myeloma (ENDEAVOR): a randomised, phase 3, open-label, multicentre study. Lancet Oncol. 2016;17(1):27-38. 